\title{
Crystallization kinetics of palm oil in blends with palm-based diacylglycerol
}

\begin{abstract}
Crystallization kinetics of palm oil (PO) in the presence of different concentrations $(2,5,10$, 30 and, $50 \% \mathrm{w} / \mathrm{w}$ ) of palm-based diacylglycerol (PB-DAG) were investigated over different ranges of crystallization temperatures. Addition of 30 and $50 \%(\mathrm{w} / \mathrm{w})$ of PB-DAG (high concentrations) increased significantly $(\mathrm{P}<0.05)$ the melting point and crystallization onset while addition of 2 and 5\% PB-DAG did not have significant $(\mathrm{P}>0.05)$ effect. PO and PO blends with 2 and $5 \%$ of PB-DAG showed crystal transformation at crystallization temperatures (TCr) of $26,26,26.5^{\circ} \mathrm{C}$, respectively as reflected in corresponding changes of the Avrami parameters at below and above these TCr. This was especially evident for the blends containing 2 and 5\% of PB-DAG. Individual comparison of induction time (Ti), Avrami exponent (n), Avrami constant (k) and half-time of crystallization (t1/2) of blends classified under various supercooling ranges based on the supercooling closeness $\left( \pm 0.1^{\circ} \mathrm{C}\right)$, showed that addition of 5\% of PB-DAG in most of the supercooling ranges significantly $(\mathrm{P}<$ $0.05)$ reduced nucleation rate as well as crystal growth velocity of PO. This was reflected in the significantly $(\mathrm{P}<0.05)$ higher $\mathrm{Ti}$ and $\mathrm{t} 1 / 2$ and lower $\mathrm{k}$. Although the presence of $2 \%$ of PB-DAG was found to have inhibitory effect on PO crystallization, this effect was not significant $(\mathrm{P}>0.05)$. Mode of crystal growth attributed to $\mathrm{n}$ was changed significantly only in presence of $5 \%$ of PB-DAG. Furthermore, presence of $10 \%$ PB-DAG showed $\beta^{\prime}-$ stabilizing effect on PO. On the other hand, high concentrations of PB-DAG were found to significantly $(\mathrm{P}<0.05)$ reduce $\mathrm{Ti}$ as well as $\mathrm{t} 1 / 2$ and also increase $\mathrm{k}$ suggesting their promoting effects on nucleation and crystallization rate of $\mathrm{PO}$ even with the close supercoolings. In addition, they changed crystal growth mode of PO. Amongst the different concentrations of PB-DAG investigated, blend containing 50\% of PB-DAG as compared to PO, not only, have healthier benefits but also, may have greater potential applications in plastic fat products due to its unique physical properties.
\end{abstract}

Keyword: Palm oil; Palm-based diacylglycerol; Isothermal crystallization; Avrami model 